\title{
Orbital-attitude coupling effects on the motion of a dumbbell space tether
}

\author{
Christopher Murray* \\ Clyde Space Ltd, \\ Sky Park 5, 45 Finnieston St., \\ Glasgow, G3 8JU, Scotland, UK \\ Email: Chris.Murray@clyde-space.com \\ *Corresponding author
}

\section{Matthew P. Cartmell}

\author{
Department of Mechanical Engineering, \\ University of Sheffield, \\ Sir Frederick Mappin Building, Mappin St., \\ Sheffield, S1 3JD, England, UK \\ Email: M.Cartmell@sheffield.ac.uk
}

\begin{abstract}
The effects of the divergence between the centre of mass and centre of gravity on the orbital motion of a dumbbell space tether, treated as a satellite of finite size, are investigated. For a radially aligned dumbbell tether with the angular speed required for a circular orbit of its centre of mass; the centre of mass of the system will traverse a trajectory from orbital apogee to perigee. This is found to be a direct result of this mass and gravity centre divergence and the system only undertakes a circular orbit when it has the angular speed required for a circular orbit of a particle at its centre of orbit. The coupling between the orbital and attitude motion for a radially aligned dumbbell tether is investigated and it is shown that for small deviations from the angular speed required for a circular orbit a libration of the tether spans is generated. This can progress to spin if the angular speed is increased further. Furthermore, it is shown that tether spans with a small initial speed relative to the centre of mass result in significant variations in orbital motion of the system. Finally, the exchange of energy between the orbital and attitude motion is investigated.
\end{abstract}

Keywords: space tethers; momentum exchange; dumbbell tether; orbital-attitude coupling; orbital dynamics.

Reference to this paper should be made as follows: Murray, C. and Cartmell, M.P. (2015) 'Orbital-attitude coupling effects on the motion of a dumbbell space tether', Int. J. Space Science and Engineering, Vol. 3, No. 2, pp.93-112.

Biographical notes: Christopher Murray is an Engineer at Clyde Space Ltd in Glasgow. He has a PhD from the University of Glasgow in the astrodynamics of space tethers.

Matthew P. Cartmell is a Professor at the University of Sheffield. He has a PhD from the University of Edinburgh in nonlinear dynamics. 


\section{Introduction}

A satellite of finite dimensions cannot be treated as a point mass, located at its centre of mass, undertaking a Keplerian orbit about the attracting body. This is due to the deviation between the centre of mass of the satellite and its centre of gravity. According to Rimrott (1989a), the location of the centre of gravity of a satellite of finite dimensions does not coincide with its centre of mass. The location of the centre of gravity is not fixed within the body and is determined by the attitude of the satellite and the distance between the satellite and the attracting body. The conditions under which the satellite might undertake a circular orbit about an attracting body were investigated by Arnold (1987). It was found that, for a radially aligned dumbbell tether system, the lower mass would experience greater gravitational force than the upper mass and that the upper mass would experience a greater centrifugal force than the lower mass. The point at which these two forces balance will experience no acceleration for a circular orbit of the system. This point of balance was termed the 'centre of orbit' and lies between the centre of mass and the centre of gravity of the satellite. Therefore, the orbital angular speed required for the dumbbell satellite to undertake a circular orbit corresponds to the angular speed required for a circular orbit of a particle located at this centre of orbit. Woo and Misra (2013) showed that disregarding the distinction between the centre of mass and centre of orbit for very long tethered systems resulted in inconsistencies when attempting to satisfy boundary conditions in stress calculations.

In addition to the divergence of the centre of mass, gravity and orbit for a satellite of finite dimensions; the orbital and attitude motion of a satellite cannot be treated independently as is the case when treating a satellite as a particle located at its centre of mass. According to Beletskii (1966); if a satellite is a rigid body of finite dimensions moving under the influence of an attracting body, its orbital and attitude motions are not independent and the centre of mass undertakes a non-Keplerian trajectory. Quadrelli et al. (2004) showed that the orbital and attitude dynamics of a satellite are only uncoupled when the satellite is in a circular orbit about the attracting body. An analysis of this orbital-attitude coupling was made by Hughes (2004) and this coupling between the orbital and attitude motion has been found for satellites with extended inertia distribution by Quadrelli et al. (2004). Several studies have investigated the coupling between the orbital and attitude motions of rigid bodies in orbit by means of approximations applied to the coupled orbital-attitude equations of motion. For example, Mohan et al. (1972) investigated the interaction between the attitude libration and orbital motion for orbits of low eccentricity by applying the method of averaging; Moran (1961) considered plane librations on the orbital motion of the centre of mass with the equations of motion being treated to a perturbation technique when considering the length of the satellite to be small in comparison to its orbital radius. Lange (1970) considered the coupling between the orbital and attitude motions of a rigid body by linearising the motion about a nominal circular orbit. Finally, Sincarsin and Hughes (1983) investigated orbital-attitude coupling for very large spacecraft by expanding the differential gravitational force and torque in a Taylor series in a small parameter defined as the ratio of spacecraft size to orbital radius.

The aim of this paper is to investigate the orbital-attitude coupling effects on the motion about Earth of a very large space tether by means of numerical integration of the equations of motion. To achieve this, the simplest space tether model was selected. This takes the form of a dumbbell space tether and is essentially two masses connected by a long tether. The tether sub-span length, $L$, refers to the tether length connecting each of 
the end-masses to the centre of mass of the system. These tether sub-spans are considered to be fully deployed and remain at a fixed length throughout the analysis. Therefore, the dumbbell system consists of two tether sub-spans; one connecting the instantaneous lower mass to the centre of mass and the other connecting the instantaneous upper mass to the centre mass even if no actual lumped mass is located at this centre of mass. The paper begins by deriving the coupled orbital-attitude equations of motions of the system. Data is then obtained on the motion of the centre of mass of a radially aligned dumbbell tether when it has the orbital angular speed required for a circular orbit at its centre of mass. The generation of libration and spin of the tether sub-spans for varying orbital angular speeds is investigated, in addition to the effect of an initial tether rotational speed on the centre of mass motion. The effects of tether sub-span length on libration amplitude are investigated and the paper concludes with an examination of the relationship between the rotational energy of the dumbbell masses and the orbital energy of the centre of mass for the coupled orbital-attitude dumbbell tether.

\section{Equations of motion}

The equations of motion of two identical bodies connected by a massless, inextensible and rigid tether in orbit about a spherical Earth are derived. For simplicity, we consider only the force of gravity acting on the bodies and tether sub-span motion within the orbital plane of the centre of mass of the system. We define the system relative to a non-inertial body-fixed frame of reference with its origin, $o$, coincident with the centre of mass of the system relative to Earth. This centre of mass lies along the tether span mid-way between the two masses. The body frame is composed of the $\{i, j, k\}$ unit vector triad with the $i$ axis directed along the local gravity gradient with its positive direction pointing away from Earth; the $j$ axis lies perpendicular to the $i$ axis, rotated in an anti-clockwise direction; and the $k$ axis lies perpendicular to the plane formed by the $\{i, j\}$ axes.

The components of the system are transformed from the body frame into a perifocal frame of reference by a coordinate transformation. The perifocal frame was described by Vallado (2007) as follows: the origin of the frame is located at the orbital focus, in this case the centre of Earth, and is composed of the $\{P, Q, W\}$ unit vector triad. Axes $\{P, Q\}$ form the orbital plane of the motion of the centre of mass with the $P$ axis directed from the frame origin towards the orbit perigee for the system's centre of mass, coincident with the origin of the body frame; the $Q$ axis is rotated 90 degrees from the $P$ axis in the direction of motion; and the $W$ axis is normal to the orbital plane, parallel to the angular momentum vector of the system. The perifocal frame can be considered to be an inertial frame as no external forces are acting on this frame of reference as a result of considering a spherical Earth, and the force of gravity only. There is little evidence to confirm that conceptual studies of dumbbell tethers need the inclusion of higher order nonlinearities such as those emanating from the consideration of orbital perturbations such as the zonal and tesseral harmonics $J_{2}$ and $J_{22}$. It is therefore assumed that the responses obtained here without including second order acceleration terms from perturbational effects are plausible for a study at this level. The configuration of the dumbbell tether system, body frame and perifocal frame is shown in Figure 1, and the parallel $k$ and $W$ axes point out of the page to form right hand sets for the body-fixed and perifocal frames respectively. 
Figure 1 Perifocal frame, body frame and dumbbell tether system configuration

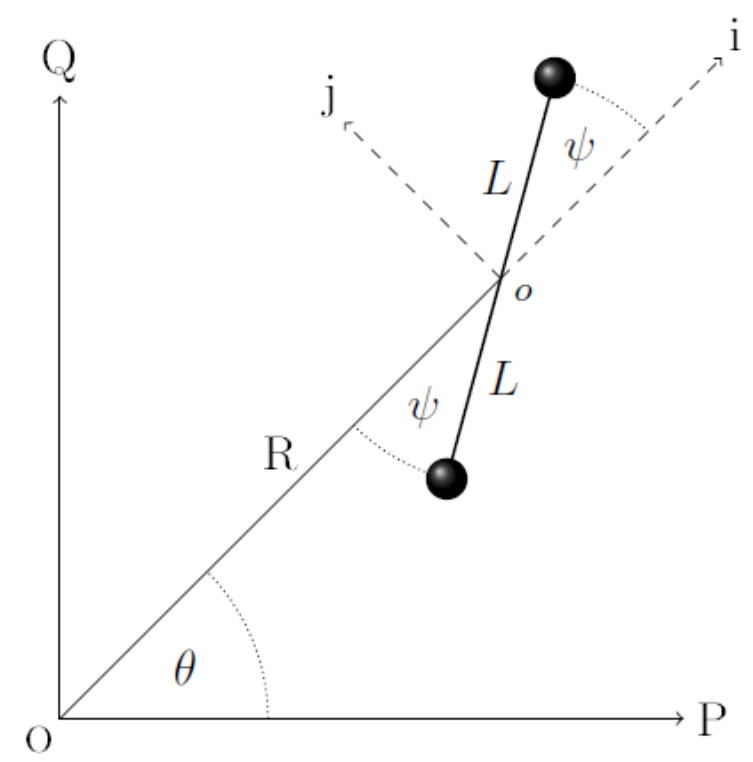

The equations of motion are derived relative to the perifocal frame using Lagrangian mechanics. Initially, the position of each dumbbell mass relative to the body frame can be specified in terms of the tether sub-span length, $L$, which is the invariant distance of each payload mass from the origin of the body frame and by the tether sub-span rotation angle, $\psi$, between the tether span and the $i$ axis of the body frame. As a result of considering only in-plane tether motion; the tether sub-span rotation angle, $\psi$, lies within the $\{i, j\}$ plane of the body frame. The position of the mass with a component initially directed along the negative $i$ axis of the body frame is termed the lower mass, $\vec{r}_{l}$, and the mass with a component initially directed along the positive $i$ axis is termed the upper mass, $\vec{r}_{u}$. The positions of the masses relative to the body frame are as follows:

$$
\vec{r}_{l}=-L\left[\begin{array}{lllll}
\cos \psi & \sin \psi & 0
\end{array}\right]^{T} \quad \vec{r}_{u}=L\left[\begin{array}{lll}
\cos \psi & \sin \psi & 0
\end{array}\right]^{T}
$$

The body frame positions of the upper and lower masses are converted into the inertial perifocal frame using the following transformation matrix (Battin, 1999):

$$
\left[T_{\theta}\right]=\left[\begin{array}{ccc}
\cos \theta & -\sin \theta & 0 \\
\sin \theta & \cos \theta & 0 \\
0 & 0 & 1
\end{array}\right]
$$

where $\theta$ is the angle between the $P$ axis of the perifocal frame and the origin of the body frame; this is termed the 'true anomaly'. The position vector of each of the masses relative to the perifocal frame, $\vec{r}_{p}$, can be found by transforming its body frame position vector, $\vec{r}_{b}$, into the perifocal frame and adding it to the position vector of the body frame's origin relative to the perifocal frame, $\vec{r}_{o}$ :

$$
\vec{r}_{p}=\vec{r}_{o}+\left[T_{\theta}\right] \vec{r}_{b}
$$


and for the body frame origin positioned at radius $R$ from the origin of the perifocal frame, we define $\vec{r}_{o}$ as:

$$
\vec{r}_{o}=R\left[\begin{array}{lll}
\cos \theta & \sin \theta & 0
\end{array}\right]^{T}
$$

The position vectors of the lower, $\vec{R}_{l}$, and upper, $\vec{R}_{u}$, masses relative to the inertial perifocal frame are therefore:

$$
\begin{aligned}
& \vec{R}_{l}=\left[\begin{array}{lll}
R \cos \theta-L \cos (\theta+\psi) & R \sin \theta-L \sin (\theta+\psi) & 0
\end{array}\right]^{T} \\
& \vec{R}_{u}=\left[\begin{array}{lll}
R \cos \theta+L \cos (\theta+\psi) & R \sin \theta+L \sin (\theta+\psi) & 0
\end{array}\right]^{T}
\end{aligned}
$$

Differentiating equations (5) and (6) with respect to time, we obtain the velocity vectors of the lower, $\vec{V}_{l}$, and upper, $\vec{V}_{u}$, masses relative to the inertial perifocal frame as:

$$
\begin{gathered}
\vec{V}_{l}=\left[\begin{array}{c}
\dot{R} \cos \theta-\dot{\theta} R \sin \theta+(\dot{\theta}+\dot{\psi}) L \sin (\theta+\psi) \\
\dot{R} \sin \theta+\dot{\theta} R \cos \theta-(\dot{\theta}+\dot{\psi}) L \cos (\theta+\psi) \\
0
\end{array}\right] \\
\vec{V}_{u}=\left[\begin{array}{c}
\dot{R} \cos \theta-\dot{\theta} R \sin \theta-(\dot{\theta}+\dot{\psi}) L \sin (\theta+\psi) \\
\dot{R} \sin \theta+\dot{\theta} R \cos \theta+(\dot{\theta}+\dot{\psi}) L \cos (\theta+\psi) \\
0
\end{array}\right]
\end{gathered}
$$

To apply Lagrangian dynamics we need the kinetic and potential energies of the dumbbell tether from its components measured relative to the perifocal frame. From equations (7) and (8) the kinetic energy of the tether system is obtained as:

$$
T=\frac{1}{2} m\left(V_{l}^{2}+V_{u}^{2}\right)=m\left(\dot{R}^{2}+\dot{\theta}^{2} R^{2}+(\dot{\theta}+\dot{\psi})^{2} L^{2}\right)
$$

with the mass of each dumbbell mass denoted by $m$. The gravitational potential energy of the tether system is obtained as:

$$
U=\frac{-\mu m}{R_{l}}+\frac{-\mu m}{R_{u}}=\frac{-\mu m}{\sqrt{R^{2}+L^{2}-2 R L \cos \psi}}+\frac{-\mu m}{\sqrt{R^{2}+L^{2}+2 R L \cos \psi}}
$$

with $\mu$ denoting the gravitational parameter of Earth. We obtain the Lagrangian of the system as:

$$
\begin{aligned}
\mathrm{L}= & T-U \\
= & m\left(\dot{R}^{2}+\dot{\theta}^{2} R^{2}+(\dot{\theta}+\dot{\psi})^{2} L^{2}\right)+\frac{\mu m}{\sqrt{R^{2}+L^{2}-2 R L \cos \psi}} \\
& +\frac{\mu m}{\sqrt{R^{2}+L^{2}+2 R L \cos \psi}}
\end{aligned}
$$


Gravity is a conservative force therefore the system's equations of motion can be obtained by applying Lagrange's equation (Fowles and Cassiday, 1993) to the system's generalised coordinates $(R, \theta, \psi)$ :

$$
\frac{d}{d t}\left(\frac{\partial \mathrm{L}}{\partial \dot{q}_{k}}\right)-\frac{\partial \mathrm{L}}{\partial q_{k}}=0 \quad(k=1,2, \ldots, n)
$$

where $q_{k}$ is the system's generalised coordinate and $\dot{q}_{k}$ is its derivative with respect to time. Applying equation (12) to the generalised coordinate for the radius of the body frame origin, $R$, we obtain the equation of motion for this coordinate:

$$
\ddot{R}-\dot{\theta}^{2} R+\frac{\mu(R-L \cos \psi)}{2\left(R^{2}+L^{2}-2 R L \cos \psi\right)^{\frac{3}{2}}}+\frac{\mu(R+L \cos \psi)}{2\left(R^{2}+L^{2}+2 R L \cos \psi\right)^{\frac{3}{2}}}=0
$$

then we obtain the equation of motion for the true anomaly, $\theta$, as:

$$
(\ddot{\theta}+\ddot{\psi}) L^{2}+\ddot{\theta} R^{2}+2 \dot{\theta} \dot{R} R=0
$$

and, finally, we obtain the equation of motion for the tether sub-span rotation angle, $\psi$, as:

$$
(\ddot{\theta}+\ddot{\psi}) L^{2}+\frac{\mu R L \sin \psi}{2\left(R^{2}+L^{2}-2 R L \cos \psi\right)^{\frac{3}{2}}}-\frac{\mu R L \sin \psi}{2\left(R^{2}+L^{2}+2 R L \cos \psi\right)^{\frac{3}{2}}}=0
$$

Nonlinear terms are evident within equations (13), (14) and (15). Additionally, the true anomaly of the centre of mass, $\theta$, and the tether sub-span rotation angle, $\psi$, are clearly coupled through their inertia terms and this results in the coupling between the orbital and attitude dynamics of the dumbbell system.

\section{Tether system orbital motion}

Having obtained the equations of motion for the system relative to the perifocal frame, we analyse the orbital motion of the dumbbell tether's centre of mass relative to the origin of this frame, coincident with the centre of Earth. This is achieved using numerical integration code written in the MATHEMATICA package. In the following analysis the tether sub-spans are all initially aligned along the local gravity gradient with zero angular speed relative to the centre of mass, $\psi_{o}=\dot{\psi}_{o}=0$.

The first case to be analysed is for a centre of mass radius of 7,500 km from Earth; a tether sub-span length of $100 \mathrm{~km}$; and payload masses of $1,000 \mathrm{~kg}$ each. The initial orbital angular speed of the system is consistent with that of a circular orbit at the radius of the centre of mass from Earth, in this case $\dot{\theta}_{o}=0.972023 \mathrm{mrad} / \mathrm{s}$, and is shown in Figure 2(a). The second case is identical to the first other than with an increase in tether 
sub-span length from $100 \mathrm{~km}$ to $200 \mathrm{~km}$ and this is shown in Figure 2(b). In Figure 2, the dashed lines represent the orbital radius of a particle initially coincident with the centre of mass with an identical orbital angular speed. Additionally, the solid line is the orbital radius of the centre of mass of the system.

Figure 2 Centre of mass orbital variation for (a) $100 \mathrm{~km}$ and (b) $200 \mathrm{~km}$ tether sub-span lengths

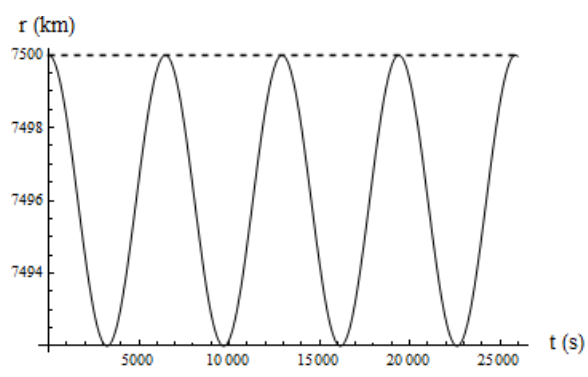

(a)

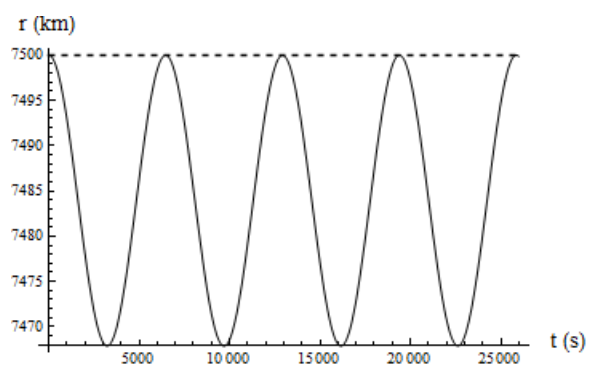

(b)

In Figure 2(a), the orbital motion of the $100 \mathrm{~km}$ sub-span system's centre of mass deviates from the circular motion of a particle initially coincident with this centre of mass. Beginning at an orbital radius of $7,500 \mathrm{~km}$, the centre of mass moves in an elliptical orbit to its closest approach to Earth which occurs at a radius of 7,492 $\mathrm{km}$. In the terminology of an Earth orbiting satellite: the centre of mass motion begins at an apogee radius of $7,500 \mathrm{~km}$ and traverses a trajectory to its perigee at 7,492 $\mathrm{km}$ from Earth. In Figure 2(b), the increase in sub-span length from $100 \mathrm{~km}$ to $200 \mathrm{~km}$ has decreased the perigee distance of the tether relative to Earth. Again, the centre of mass begins at an apogee distance of 7,500 $\mathrm{km}$ but in this case has a perigee distance of 7,468 $\mathrm{km}$. In both cases, the motion of the centre of mass from an initial apogee to its perigee is evidence that the tether has insufficient orbital speed, and therefore orbital energy, to remain on a circular orbit about Earth.

From the cases shown, it is evident that the displacement of the payload masses from the centre of mass results in a deviation in the orbit of the centre of mass from that of an initially coincident particle with identical initial conditions. This results in a reduction in the orbital radius in both cases and it is evident that increasing this mass displacement, by an increase in sub-span length, increases the magnitude of this orbital deviation. The resulting motion of the centre of mass is consistent with the analysis given by Arnold (1987) which found that a satellite of finite dimensions will only undertake a circular orbit at the angular speed required for a circular orbit of a particle located at the centre of orbit. Rimrott (1989a) showed that the centre of gravity for a radially aligned rod satellite lies closer to the point of gravitational attraction than the centre of mass. For a dumbbell tether system the centre of orbit therefore lies at a radius closer to Earth than its centre of mass. Therefore, a radially aligned dumbbell tether with the orbital angular speed for a circular orbit at the centre of mass will undertake a trajectory from apogee to perigee due to its angular velocity being smaller than that required to undertake a circular orbit about the Earth. 


\section{Tether sub-span libration generation due to orbital angular speed}

To explore the effects of this coupling within the equations of motion; we analyse the attitude motion of the sub-spans as the orbital angular speed deviates from that required for a circular orbit of a particle located at the centre of orbit. The angular speed required for a circular orbit at the centre of orbit is obtained by setting the sub-span rotation angle equal to zero in equation (13) and solving for $\dot{\theta}$. This is obtained as:

$$
\dot{\theta}=\sqrt{\frac{\mu\left(R_{l}^{2}+R_{u}^{2}\right)}{R_{l}^{3} R_{u}^{2}+R_{l}^{2} R_{u}^{3}}}
$$

Contrary to the definition of libration as vibratory motion superimposed onto locked rotary, or orbital, motion as given by Rimrott (1989b), we will consider libration as defining the attitude motion of the sub-spans relative to the body fixed frame oscillating about the centre of mass. In the following the tether sub-spans are initially aligned along the local gravity gradient with zero relative speed.

We begin by examining the motion of the tether sub-spans when the system has the orbital angular speed required for a circular orbit of a particle located at the centre of orbit. The initial conditions are a centre of mass radius of 7,500 km and a tether sub-span length of $100 \mathrm{~km}$. The required orbital angular speed for a circular orbit is calculated to be $\dot{\theta}=0.972283 \mathrm{mrad} / \mathrm{s}$, shown in Figure 3(a). It is clear that no libration of the tether sub-spans is generated and they remain aligned in a locked configuration along the local gravity gradient throughout the orbital motion. Figure 3(b) shows the rotation angle of the sub-spans given the orbital angular speed for a circular orbit at its initial centre of mass position using the initial conditions of Figure 2(a). In contrast to the case of Figure 3(a), small aperiodic librations are found to be generated with a maximum amplitude of 0.836 mradians even for small variations in the orbital angular speed from that required for a circular orbit of the system. Interestingly, the initial motion of the tether is in the retrograde direction as the system moves from its apogee radius of 7,500 km to its perigee.

Figure 3 Tether sub-span libration generation with varying orbital angular speed 1, (a) tether rotation for a circular orbit of system (b) tether rotation for a circular orbit at centre of mass

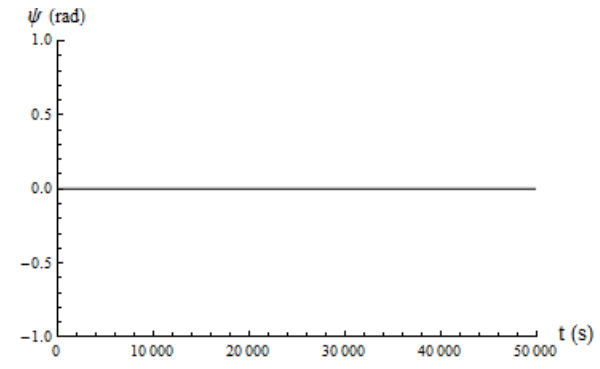

(a)

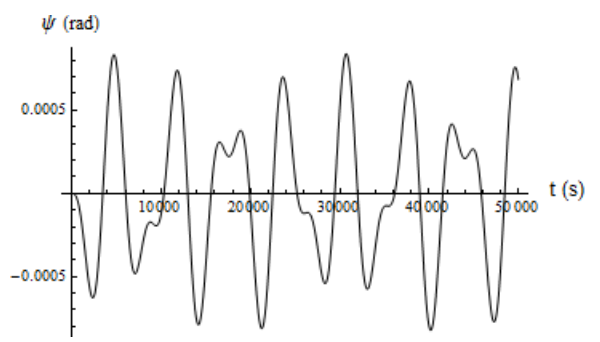

(b) 
Figure 4 Tether sub-span libration generation with varying orbital angular speed 2, (a) tether rotation for decreased angular speed (b) tether rotation for increased angular speed

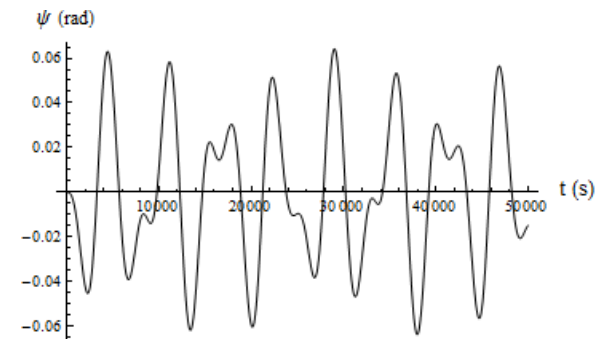

(a)

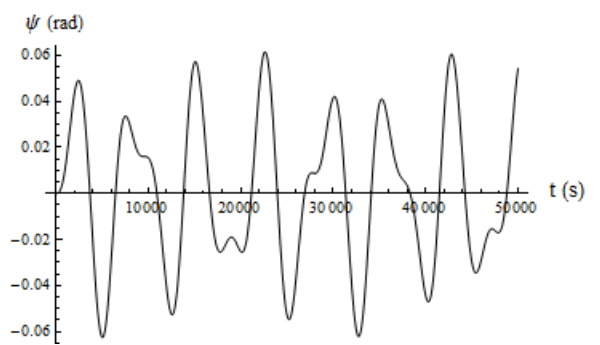

(b)

Figures 4(a) and 4(b) show the sub-span motion when the orbital angular speed is decreased and increased, respectively. Figure 4(a) shows the sub-span motion when the initial orbital angular speed is reduced to that required for the circular orbit of a particle located at 7,600 km, $\dot{\theta}_{o}=0.953 \mathrm{mrad} / \mathrm{s}$, whilst Figure 4(b) shows the sub-span motion when the angular speed is increased to that required for the circular orbit of a particle located at $7,400 \mathrm{~km}, \dot{\theta}_{o}=0.992 \mathrm{mrad} / \mathrm{s}$. In both cases the libration amplitudes have increased, in comparison to those generated for a circular orbit at the centre of mass, whilst remaining aperiodic. The maximum amplitude of both librations is 0.063 radians with the motion in Figure 4(a) beginning with a retrograde rotation whilst that in Figure 4(b) begins with a prograde rotation.

Figure 5 Tether sub-span libration generation with varying orbital angular speed 3, (a) tether rotation for further increased angular speed (b) induced tether spinning for increased angular speed

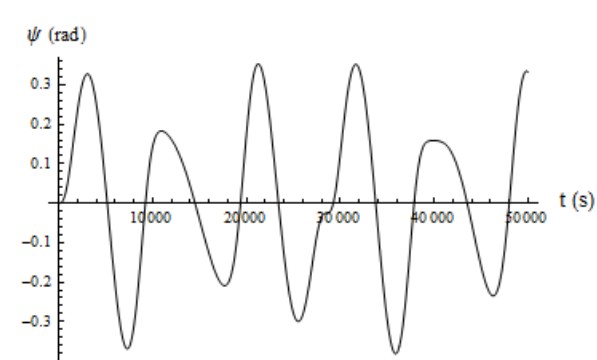

(a)

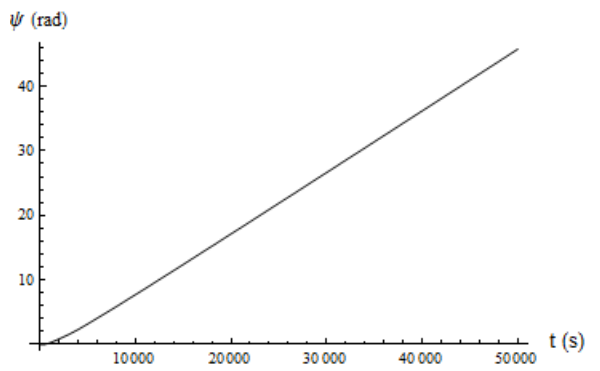

(b)

Figures 5(a) and 5(b) show the sub-span motion when the orbital angular speed of the tether has been increased further. Figure 5(a) shows the sub-span motion when the angular speed is increased to that required for the circular orbit of a particle located at $7,000 \mathrm{~km}, \dot{\theta}_{o}=1.078 \mathrm{mrad} / \mathrm{s}$, whilst Figure 5(b) shows the sub-span motion when the angular speed has increased to $\dot{\theta}_{o}=1.3584441 \mathrm{mrad} / \mathrm{s}$. The latter places the system in a highly elliptical orbit about Earth. In Figure 5(a), the maximum amplitude of the tether libration has increased to 0.3488 radians as a result of the increased orbital angular speed of the system whilst the motion remains aperiodic. Further increasing the orbital angular 
speed, as in Figure 5(b), generates a spinning motion of the sub-spans about the centre of mass. Between the conditions of libration and spin is a transitional phase for the tether which consists of periods of spin interspersed with periods of libration which have not been shown here.

In general, for initially gravity gradient aligned tether sub-spans, tether libration in systems which begin their motion from apogee to perigee are initially retrograde whilst those going from perigee to apogee are initially prograde. This is a direct result of the coupling between the orbital and attitude motion. As the system moves from apogee to perigee the radial speed of its orbital motion is negative and so the sub-spans experience a Coriolis acceleration, evident within the equations of motion, and this opposes orbital motion. This results in an initial retrograde trajectory for the radially aligned sub-spans. For motion from perigee to apogee the converse is true, the orbital radial speed in this case is positive and the tether experiences a Coriolis acceleration in the same direction as the orbital motion resulting in a prograde libration of the radially aligned sub-spans.

From the data accumulated it has been shown that for any orbital angular speed other than that required for a circular orbit of the system, obtained from equation (16), a libration is generated in the tether sub-spans and this can progress to spin as the initial orbital speed of the system is varied. In real world applications this has many consequences, for example; in a symmetrically laden momentum exchange tether configuration any extension of the tether sub-spans when the system is in an initial circular orbit will result in libration of the tether sub-spans. This is due to a change in the radius of the centre of orbit with tether extension: the circular orbit angular speed at the centre of orbit being unequal to that required for a circular orbit of the final centre of orbit location. Consequently, the offset in orbital angular speed generates a libration in the tether sub-spans. Another example may be the deployment of a tether from an orbiting satellite; so even if the satellite has an initial angular speed necessary for a circular orbit at the centre of orbit of the final configuration, and this speed is maintained throughout deployment, libration will occur as a result of the system passing through the varying orbital configurations required to achieve the final state.

\section{The effect of initial tether sub-span speed on the centre of mass motion}

It has been shown that due to the coupling between the orbital and attitude motion of the dumbbell system a deviation from the orbital angular speed required for a circular orbit at the centre of orbit will result in an alteration in the attitude motion of the sub-spans. For small deviations this results in the generation of tether sub-span libration. Increasing this deviation results in the transition from tether libration to spin. We now examine the effect that the attitude motion has on the orbital motion by giving the tether sub-spans an initial rotational speed about the centre of mass. To examine this we compare the orbital motion of the centre of mass of two identical tethers both with the orbital angular speed required for a circular orbit at the centre of orbit, which we obtain from equation (16), and both initially aligned along the local gravity gradient. The only difference between the systems is that one system has zero sub-span rotational speed and the other has an initial sub-span rotational speed, $\dot{\psi}_{o}$, which results in either libration; quasi-periodic motion; or spinning of the sub-spans. 
Using an initial centre of mass radius for both systems of 7,500 km and payload masses of $1,000 \mathrm{~kg}$ each; we examine the motion for a $50 \mathrm{~km}$ tether sub-span length from which we obtain the required speed for a circular orbit as $\dot{\theta}_{o}=0.9721 \mathrm{mrad} / \mathrm{s}$. The analysis begins with a small initial speed for the sub-span rotation taken as $0.01 \dot{\theta}_{o}$, where $\dot{\theta}_{o}$ is the initial orbital angular speed of the tether. This speed is in the positive, prograde, direction. The motion of the system in a locked circular orbit; the librating tether; and the rotation angle of the librating sub-spans are all shown in Figure 6.

Figure 6 (a) Centre of mass orbital radius and (b) prograde librating tether sub-span rotation angle, $\dot{\psi}_{o}=0.01 \dot{\theta}_{o}$

Librating and static tether centre of mass radii

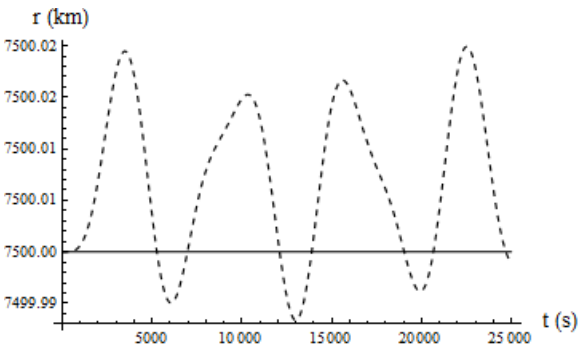

(a)

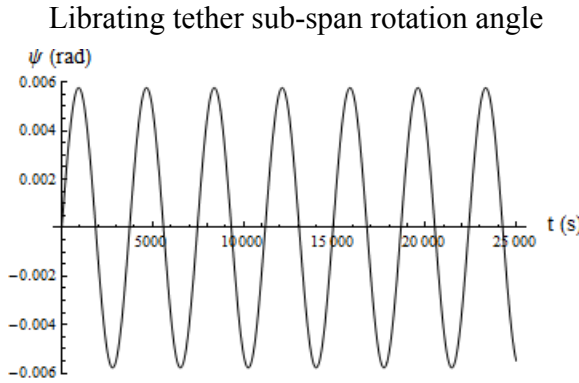

(b)

In Figure 6(a) the solid line represents the motion about Earth of the tether system in a locked circular orbit whilst the dashed line represents the motion of the centre of mass of the librating tether system about Earth. From this it is evident that a small initial speed, resulting in libration of the tether sub-spans, will affect the orbital motion of the centre of mass and in this case the orbital motion is aperiodic. The maximum deviation of the centre of mass from the circular orbit is $20 \mathrm{~m}$ and from this it can be noted that the initial prograde rotation of the sub-spans has the effect of raising the mean orbital radius above that of the initial circular orbit. The libration of the sub-spans is shown in Figure 6(b) and this is seen to be periodic with a magnitude of 5.75 mradians.

Figure 7 (a) Centre of mass orbital radius and (b) retrograde librating tether sub-span rotation angle, $\dot{\psi}_{o}=-0.01 \dot{\theta}_{o}$

Librating and static tether centre of mass radii

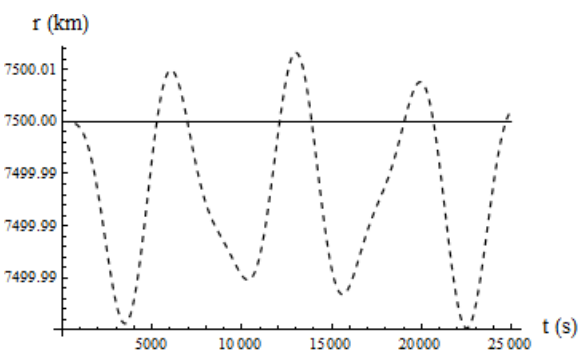

(a)
Librating tether sub-span rotation angle

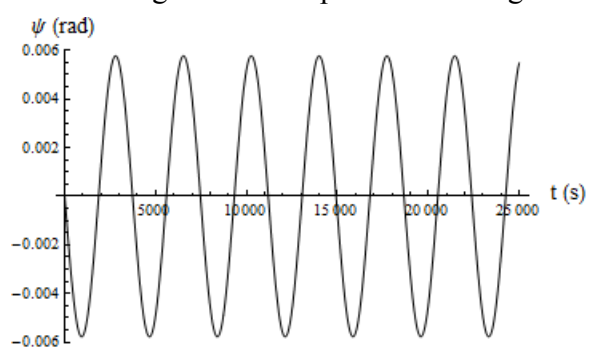

(b) 
These effects are reversed if the sub-spans are given an initial retrograde sub-span speed. In Figure 7(a), it can be seen that the orbital motion of the centre of mass of the tether deviates from the circular orbit by a maximum of $10 \mathrm{~m}$, it is also aperiodic, and the retrograde rotation has the effect of lowering the mean orbital radius of the centre of mass below that of the circular orbit. The libration of the sub-spans is shown, in Figure 7(b), and is seen to librate periodically with an amplitude of 5.75 mradians but is out of phase by $\pi$ radians with the libration in Figure 6(b).

Figure 8 (a) Centre of mass orbital radius and (b) prograde librating tether sub-span rotation angle, $\dot{\psi}_{o}=\dot{\theta}_{o}$

Librating and static tether centre of mass radii

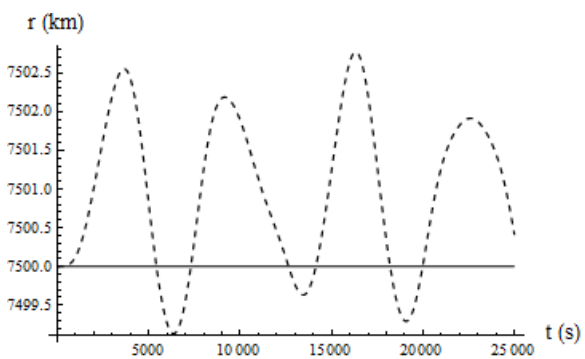

(a)
Librating tether sub-span rotation angle

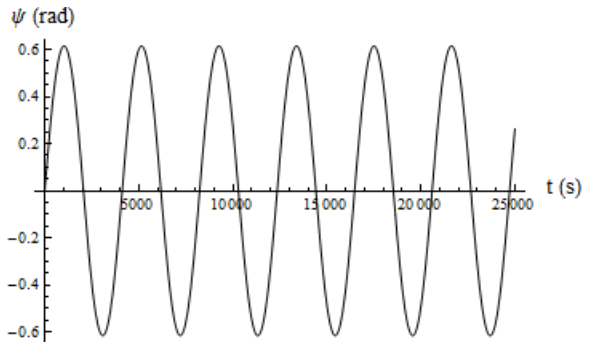

(b)

Increasing the initial speed of the prograde rotating tether to be equivalent to the initial orbital speed, $\dot{\theta}_{o}$, gives the data shown in Figure 8. In Figure 8(a), it is clear that the maximum deviation from the circular orbit has increased to $2.8 \mathrm{~km}$ as a result of the increased prograde speed of the sub-spans and again the effect of the prograde rotation is to raise the mean orbital radius above that of the circular orbit. In Figure 8(b), due to the increase in rotation speed an increase in the libration angle is evident which is again periodic with an amplitude of 0.61 radians.

Figure 9 (a) Centre of mass orbital radius and (b) prograde librating tether sub-span rotation angle, $\dot{\psi}_{o}=1.735 \dot{\theta}_{o}$

Librating and static tether centre of mass radii

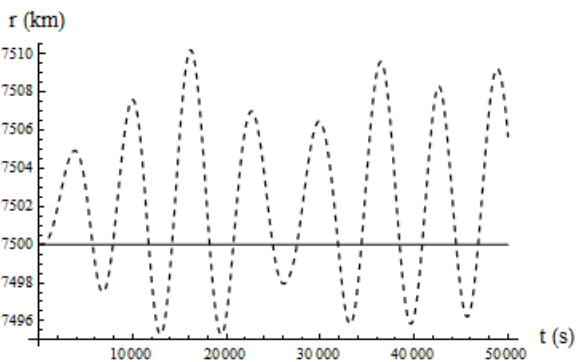

(a)

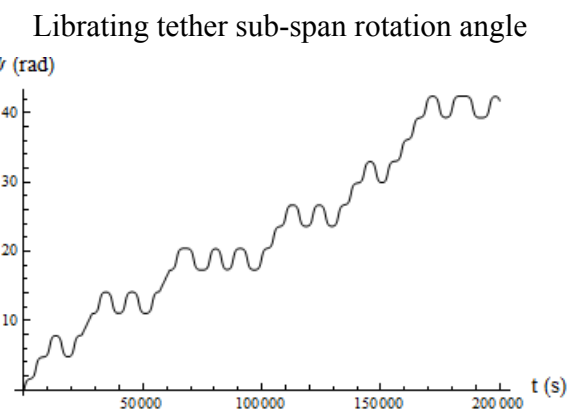

(b)

A further increase in the initial prograde speed of the sub-spans to an initial speed marginally smaller than that required to undertake pure spinning motion results in quasi-periodic motion of the tether. This occurs at an initial prograde speed of $1.735 \dot{\theta}_{o}$ 
for the initial conditions used here. The orbital radius of the centre of mass and the rotation of the sub-spans are shown in Figure 9. From Figure 9(b), the subs-spans are undergoing periods of spin interspersed with periods of libration which we term quasiperiodic. The effect of this tether motion on the orbital radius of the centre of mass is to render the centre of mass motion more uniform as shown in Figure 9(a) with an increase in the deviation of the motion from the circular orbit, this takes a maximum value of $11 \mathrm{~km}$.

By increasing the initial prograde rotation to $5 \dot{\theta}_{o}$, as shown in Figure 10, it is evident that the tether is spinning about the centre of mass. The spinning of the tether has the effect of rendering the motion of the centre of mass stable and periodic as shown in Figure 10(a). Interestingly, the maximum deviation in the orbital radius has reduced to $1.8 \mathrm{~km}$ with the system returning to the radius of the circular orbit once per orbital period coinciding with the perigee of the system's motion.

Figure 10 (a) Centre of mass orbital radius and (b) prograde librating tether sub-span rotation angle, $\dot{\psi}_{o}=5 \dot{\theta}_{o}$

Librating and static tether centre of mass radii

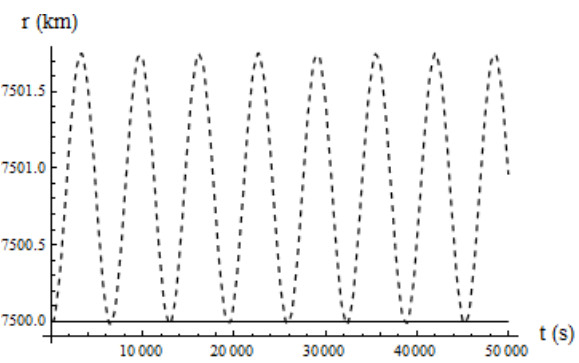

(a)
Librating tether sub-span rotation angle

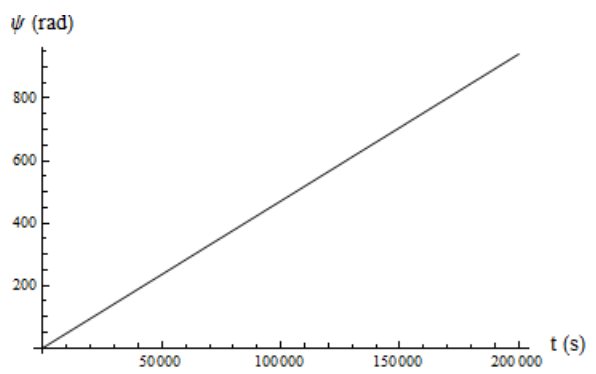

(b)

To summarise our findings; there is a complex interaction between the libration or spin of the tether, resulting from an initial tether sub-span speed, and their effects upon the orbital motion of the centre of mass. Tether libration results in aperiodic centre of mass orbital motion and an initial prograde speed of the sub-spans has the net effect of raising the mean orbital radius above that of a tether system in a circular orbit, whilst an initial retrograde speed does the opposite. Increasing the amplitude of the tether libration by means of increased initial sub-span rotational speed increases the aperiodicity of the centre of mass motion, in addition to increasing its deviation from its initial orbit. Additionally, the tether system's centre of mass only undergoes purely periodic motion when it either has zero initial sub-span speed or when it has sufficient initial speed to undertake pure spinning motion. Spinning motion may be attributed to some degree to the variation of the centre of gravity as the sub-spans rotate about the centre of mass. According to Rimrott (1989a), the centre of gravity for a tangentially orientated rod satellite lies further from the centre of attraction than does its centre of mass. Dependent upon the attitude of the rod, the centre of gravity can be seen to prescribe an arc about the centre of mass. Applying this to a tether spinning about the centre of mass, the centre of gravity and therefore centre of orbit will rotate periodically with the tether rotation rate about the centre of mass thus rendering the orbital motion more uniform than is the case with libration. 
For real world applications the above analysis shows that any spin up or spin down of fully extended tether sub-spans in orbit will affect the motion of the centre of mass. In section 7 an explanation is given in terms of the total energy of the system of why the orbit of the centre of mass is raised or lowered dependent upon the direction of the sub-spans' initial rotational speed.

\section{Variation in tether libration amplitude with sub-span length}

Having determined that libration/spin is generated within the tether for deviations in the orbital angular speed from that required for a circular orbit at the centre of orbit; we now consider the effect that the length of the sub-spans has on the magnitude of these librations, and whether a significant increase in length can result in a transition from libration into spin. We consider a centre of mass radius of 10,000 km from Earth; payload masses of 1,000 kg each; and an initial orbital angular speed of the system consistent with that required for a circular orbit at the centre of mass, in this case $\dot{\theta}_{o}=0.631348 \mathrm{mrad} / \mathrm{s}$. Once again, the tether sub-spans are considered to be initially aligned along the local gravity gradient with zero speed relative to the centre of mass.

Figure 11 Libration amplitude variation with increasing tether sub-span length

$50 \mathrm{~km}$ tether sub-span length

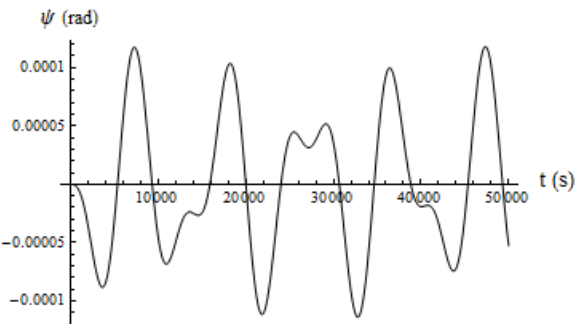

(a)

$1,000 \mathrm{~km}$ tether sub-span length

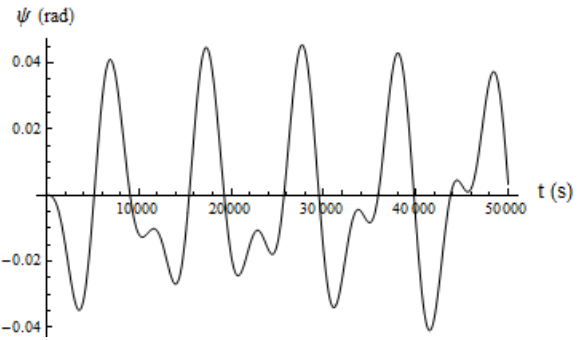

(c)

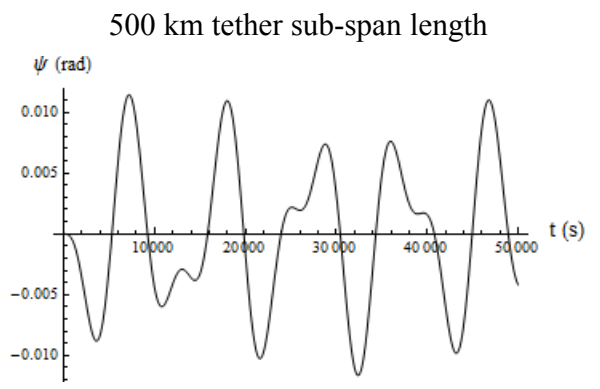

(b)

2,000 km tether sub-span length

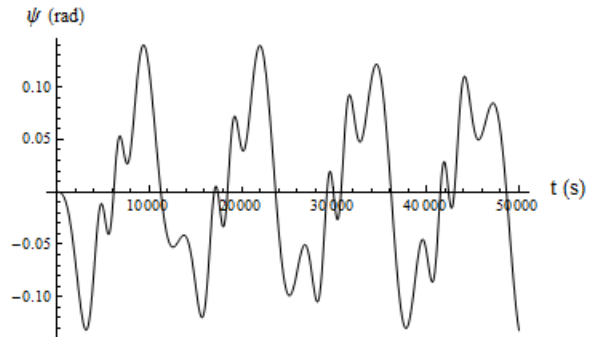

(d)

Figure 11 shows the libration generated in the tether sub-spans for lengths of 50, 500, 1,000 and 2,000 km using identical initial conditions. From this it is clear that as the tether sub-span lengths increase the amplitude of the librations generated within the system increase and in all cases their motion is nonlinear. These libration amplitudes vary nonlinearly with tether sub-span length for example, increasing the tether sub-span length 
by a factor of 10 , from $50 \mathrm{~km}$ to $500 \mathrm{~km}$, increases the maximum libration amplitude by approximately a factor of 100 . Additionally, quadrupling the tether sub-span length from $500 \mathrm{~km}$ to $2,000 \mathrm{~km}$ produces an increase in libration angle by a factor of 10 . This increase in libration amplitude can be attributed to the increasing separation between the centre of mass and centre of orbit with increasing sub-span length. Although it is evident from the analysis that increasing the sub-span length increases the magnitude of the libration angle it seems unlikely that the tether sub-span lengths used in practical scenarios would be of sufficient magnitude to generate a transition from libration to spin of the tether spans about the centre of mass.

\section{System energy}

An interesting feature of the orbital-attitude motion coupling is the exchange of energy between the orbital motion of the centre of mass and the attitude motion of the tether sub-spans. As we are dealing only with the conservative gravitational force acting on the system, the total energy must remain constant. Firstly, we examine the specific orbital energy of the centre of mass and that of a particle initially coincident with this centre of mass with identical initial conditions. These initial conditions are those used to obtain the data shown in Figures 2(a) and 2(b). However, we use tether sub-span lengths of $200 \mathrm{~km}$ and $500 \mathrm{~km}$ to illustrate more clearly this exchange of energy, and this is shown in Figures 12(a) and 12(b), respectively.

Figure 12 Centre of mass specific orbital energy variation for (a) $200 \mathrm{~km}$ and (b) $500 \mathrm{~km}$ tether sub-span lengths

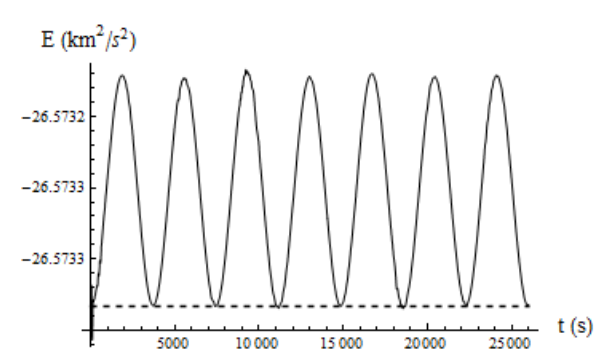

(a)

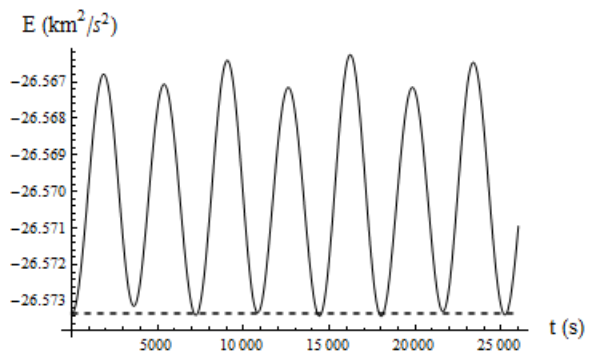

(b)

In Figures 12(a) and 12(b), the energies of both the particle moving in a circular orbit (dashed) and that of the centre of mass (solid) are shown. Notably, the initial specific energy of the centre of mass equals that of a particle located at the initial centre of mass in both cases. This is at the instant that the tether sub-spans are initially aligned along the local gravity gradient with zero rotational speed relative to the centre of mass. However, an increase in orbital energy of the centre of mass is found in both the $200 \mathrm{~km}$ and $500 \mathrm{~km}$ cases with a greater increase occurring for $500 \mathrm{~km}$. This result can be explained by noting that the orbital angular speed required for a particle to undertake a circular orbit at the system's centre of mass is lower than that required for the system to undertake a circular orbit and, as noted in Section 4 , the tether sub-spans of a system with decreased orbital angular speed will begin their motion with a retrograde libration as the body moves from apogee to perigee. As the total energy of the system remains constant; the 
centre of mass has acquired a positive energy from the retrograde rotation of the sub-spans therefore, relative to Earth, the orbital energy of the centre of mass has increased whilst that of the upper and lower masses has decreased. The consequence of this retrograde rotation is to raise the orbital perigee of the system in comparison to that which would have been obtained had the system remained aligned with the local gravity gradient throughout. However, due to the additional gravitational acceleration experienced by the tether there is a net decrease in the centre of mass motion in comparison to that of a particle located at the centre of mass.

An additional point of note is that as the system passes through perigee the energy of the centre of mass begins to decrease as the sub-spans begin to rotate in the prograde direction and in the $200 \mathrm{~km}$ case the orbital energy of the centre of mass returns to its initial value as the system returns to apogee. As noted above, the energy increase is greater in the $500 \mathrm{~km}$ sub-span system compared to the energy increase in the $200 \mathrm{~km}$ sub-span case. This is a result of the increased tether sub-span length achieving larger libration angles relative to the local gravity gradient, as obtained in Section 6, which results in an increase in the energy imparted to the centre of mass motion.

We are now going to use the equation of total energy of the system and rearrange it into its constituent parts comprising the orbital energy of the centre of mass and energy of the payload masses relative to the centre of mass. We obtain the total energy of the tether system as the sum of equations (9) and (10) as:

$$
E=m\left(\dot{R}^{2}+\dot{\theta}^{2} R^{2}+(\dot{\theta}+\dot{\psi})^{2} L^{2}\right)+\frac{-\mu m}{\sqrt{R^{2}+L^{2}-2 R L \cos \psi}}+\frac{-\mu m}{\sqrt{R^{2}+L^{2}+2 R L \cos \psi}}
$$

Rearranging equation (17) in terms of the total energy of the centre of mass, where $M$ is the total mass of the system and equivalent to $2 m$, we obtain:

$$
\begin{aligned}
E= & \frac{M}{2}\left(\dot{R}^{2}+\dot{\theta}^{2} R^{2}\right)-\frac{\mu M}{R}+m(\dot{\theta}+\dot{\psi})^{2} L^{2}+\left(\frac{-\mu m}{\sqrt{R^{2}+L^{2}-2 R L \cos \psi}}+\frac{\mu m}{R}\right) \\
& +\left(\frac{-\mu m}{\sqrt{R^{2}+L^{2}+2 R L \cos \psi}}+\frac{\mu m}{R}\right)
\end{aligned}
$$

The first two terms of equation (18) comprise the kinetic and the potential energies of the centre of mass, respectively, the sum of which can be considered to be the orbital energy. The third term is the kinetic energy arising from the rotation of the tether masses relative to the centre of mass whilst the fourth and fifth terms result from the variation in potential energy of the masses from the centre of mass, the sum of these three terms is the attitude energy.

To illustrate this exchange of energy between the orbit and attitude the variation can be shown in both, in the following example. We use an initial centre of mass radius of $7,500 \mathrm{~km}$; payload masses of 1,000 kg each; tether sub-span lengths of $500 \mathrm{~km}$; and an initial centre of mass orbital angular speed consistent with a circular orbit of a particle located at $7,000 \mathrm{~km}, \dot{\theta}_{o}=1.078 \mathrm{mrad} / \mathrm{s}$. The energy of the orbit and of the attitude is shown in Figure 13. From Figures 13(a) and 13(b) it is evident that the orbital and attitude energies are varying inversely with one another whilst the total energy of the system of $-41,109.3 \mathrm{kgkm}^{2} / \mathrm{s}^{2}$ remains constant. As the motion begins from perigee to 
apogee, the system begins with a greater angular speed than is required for a circular orbit and the orbital energy decreases as the attitude energy of the sub-spans increases due to prograde rotation. Throughout the motion there is a constant exchange between orbital and attitude energy dependent upon the motion of the tether sub-spans. From this it is clear that the orbital motion and attitude motion are inextricably linked and variation in one inherently affects the other.

Figure 12 Comparison between (a) orbital and (b) attitude energy

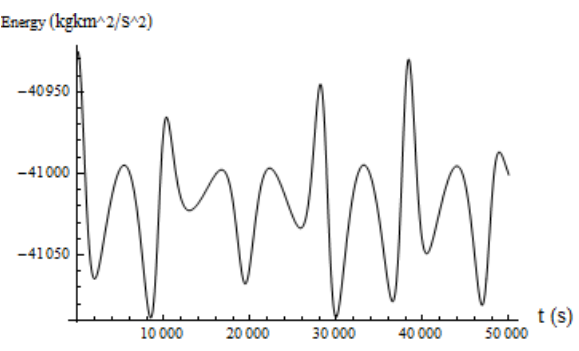

(a)

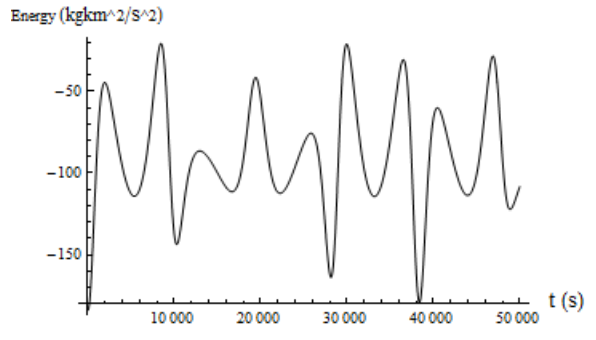

(b)

We conclude with an explanation of the raising and lowering of the centre of mass orbit due to an initial rotational speed of the sub-spans in terms of energy exchange, as noted in Section 5. The reason why the orbital radius of the centre of mass increases for an initial prograde rotation and decreases for an initial retrograde rotation is very simple: as the tether sub-spans are given an initial rotational speed relative to the centre of mass, the total energy of the system is either increased or decreased dependent upon whether the rotation is in the prograde or retrograde direction, respectively. For the prograde case, the total energy of the system is greater than that required for a circular orbit due to the direction of rotational speed. As the sub-spans move away from radial alignment in the prograde direction they experience an opposing torque due to the gravity gradient. This decelerates the sub-spans and they reach their maximum libration amplitude. The subspans now begin to move in a retrograde direction towards radial alignment which results in a net decrease in the attitude energy. As we are dealing with a conservative system, the total energy must remain constant therefore the excess attitude energy that the sub-spans initially had is contributed to the orbital energy thus raising its orbit. For the retrograde case, the total energy is lower than that required for a circular orbit as a result of the direction of the rotational speed. When the sub-spans reach their maximum retrograde libration amplitude they begin to rotate in a prograde direction due to the gravity gradient torque acting on them. This prograde rotation results in an increase in attitude energy which in turn reduces the orbital energy thus resulting in a decrease in orbital radius.

\section{Conclusions}

Having derived the equations of motion for the tether system it was clear that they contained nonlinear terms and that orbital-attitude dependence resulted from the coupling of the generalised coordinates for the true anomaly and for the tether sub-span rotation angle through their respective inertia terms. Numerical integration of these equations of motion demonstrated that, as an extended body in orbit, the tether would not undertake a 
circular orbit about Earth when given the initial angular speed required for a circular orbit at its centre of mass. However, the system would undertake a circular orbit when given the angular speed required for a circular orbit at the centre of orbit. Additionally, for a radially aligned system it was shown that it would undertake a trajectory from the apogee of its orbit to its perigee when given the angular speed required for a circular orbit at its centre of mass. This resulted from the centre of orbit lying closer to Earth than the centre of mass for this attitude configuration of the dumbbell tether and consequently the angular speed of the tether under these conditions being smaller than required to remain at its current altitude relative to Earth.

Analysis of the change in attitude motion of the tether was undertaken when variations in the orbital motion were made. It was found that even small deviations from the orbital angular speed required for a circular orbit generated librations within the initially radially aligned tether. Additionally, the libration displayed possible evidence of chaotic behaviour. Increasing this deviation demonstrated that the attitude of the tether would transform from libratory motion into spinning motion about the centre of mass: this is clear evidence of strong dynamic coupling. It was noted that for tether orbits from apogee to perigee that the sub-span librations would begin with retrograde librations with the converse being true for motions from perigee to apogee. This was found to result from the sign of the orbital radial speed term occurring within the Coriolis acceleration present in the equations of motion.

Analysis of the change in orbital motion with variation in the attitude motion was also undertaken. It was found that for a tether with the required orbital angular speed for a circular orbit that libratory attitude motion resulted in aperiodic orbital motion about Earth which increased with increasing libration amplitudes. The direction of this attitude motion was also found to be significant with an initial prograde libration having the effect of raising the orbital motion in comparison to its initial circular orbit and a retrograde motion lowering it. Interestingly, when the tether sub-spans were spinning about the centre of mass it was found that the orbital motion became periodic with a small deviation from the initial circular orbit remaining. It was postulated that this may result from a variation in the location of the centre of gravity, and therefore centre of orbit, relative to the centre of mass as the tether sub-spans rotate.

An investigation into the effect of tether sub-span length variation on the libration amplitude found that with increasing sub-span length the libration amplitude would increase nonlinearly. This results from an increased separation between the centre of mass and centre of orbit with increasing tether sub-span length.

Finally, the relationship between the orbital energy and the attitude energy of the tether was investigated. The equation for the total energy of the system was rearranged into components of orbital energy and attitude energy and it was found that the total energy of the system remained constant, as expected for a conservative system, but that there was a constant exchange of energy between the orbit and attitude. This constant exchange of energy results from the inertia coupling between the generalised coordinates for the true anomaly and tether sub-span rotation angle within the equations of motion. 


\section{References}

Arnold, D.A. (1987) 'The behavior of long tethers in space', The Journal of Astronautical Sciences, Vol. 35, No. 1, pp.3-18.

Battin, R.H. (1999) 'Some basic topics in analytical dynamics', An Introduction to the Mathematics and Methods of Astrodynamics, Revised ed., pp.79-106, AIAA Education Series, Reston, VA, USA.

Beletskii, V.V. (1966) 'The relations between translational and rotational motions of a rigid body in a Newtonian force field', Motion of an Artificial Satellite About Its Centre of Mass, Mechanics of Space Flight, Vol. 67, No. 51366, pp.85-104, NASA technical translation, Israel Program for Scientific Translations, Jerusalem.

Fowles, G.R. and Cassiday, G.L. (1993) 'Lagrangian Mechanics', Analytical Mechanics, 5th ed., pp.340-373, Saunders College Publishing, Fort Worth, TX.

Hughes, P.C. (2004) 'Gravitational stabilization', Spacecraft Attitude Dynamics, pp.281-353, Dover Publications, New York.

Lange, B. (1970) 'Linear coupling between orbital and attitude motions of a rigid body', Journal of Astronautical Sciences, Vol. 18, No. 3, pp.150-167.

Mohan, S.N., Breakwell, J.V. and Lange, B.O. (1972) 'Interaction between attitude libration and orbital motion of a rigid body in a near Keplerian orbit of low eccentricity', Celestial Mechanics, Vol. 5, No. 2, pp.157-173.

Moran, J.P. (1961) 'Effects of plane librations on the orbital motion of a dumbbell satellite', ARS Journal, Vol. 31, No. 8, pp.1089-1096.

Quadrelli, M.B., Mettler, E. and Langmaier, J.K. (2004) 'Dynamics and controls of a conceptual Jovian Moon Tour Spacecraft', AIAA/AAS Astrodynamics Specialist Conference and Exhibit, AIAA 2004-4486, Providence, Rhode Island, USA.

Rimrott, F.P.J. (1989a) 'Centre of gravity', Introductory Attitude Dynamics, pp.40-75, Springer-Verlag, New York, NY, USA.

Rimrott, F.P.J. (1989b) 'Libration', Introductory Attitude Dynamics, pp.76-86, Springer-Verlag, New York, NY, USA.

Sincarsin, G.B. and Hughes, P.C. (1983) 'Gravitational orbit-attitude coupling for very large spacecraft', Celestial Mechanics, Vol. 31, No. 2, pp.143-161.

Vallado, D.A. (2007) 'Coordinate and time systems', Fundamentals of Astrodynamics and Applications, pp.135-234, Microcosm Press, Hawthorne, CA, USA and Springer, New York, NY, USA.

Woo, P. and Misra, A.K. (2013) 'Mechanics of very long tethered systems', Acta Astronautica, Vol. 87, pp.153-162. 


\section{Nomenclature}

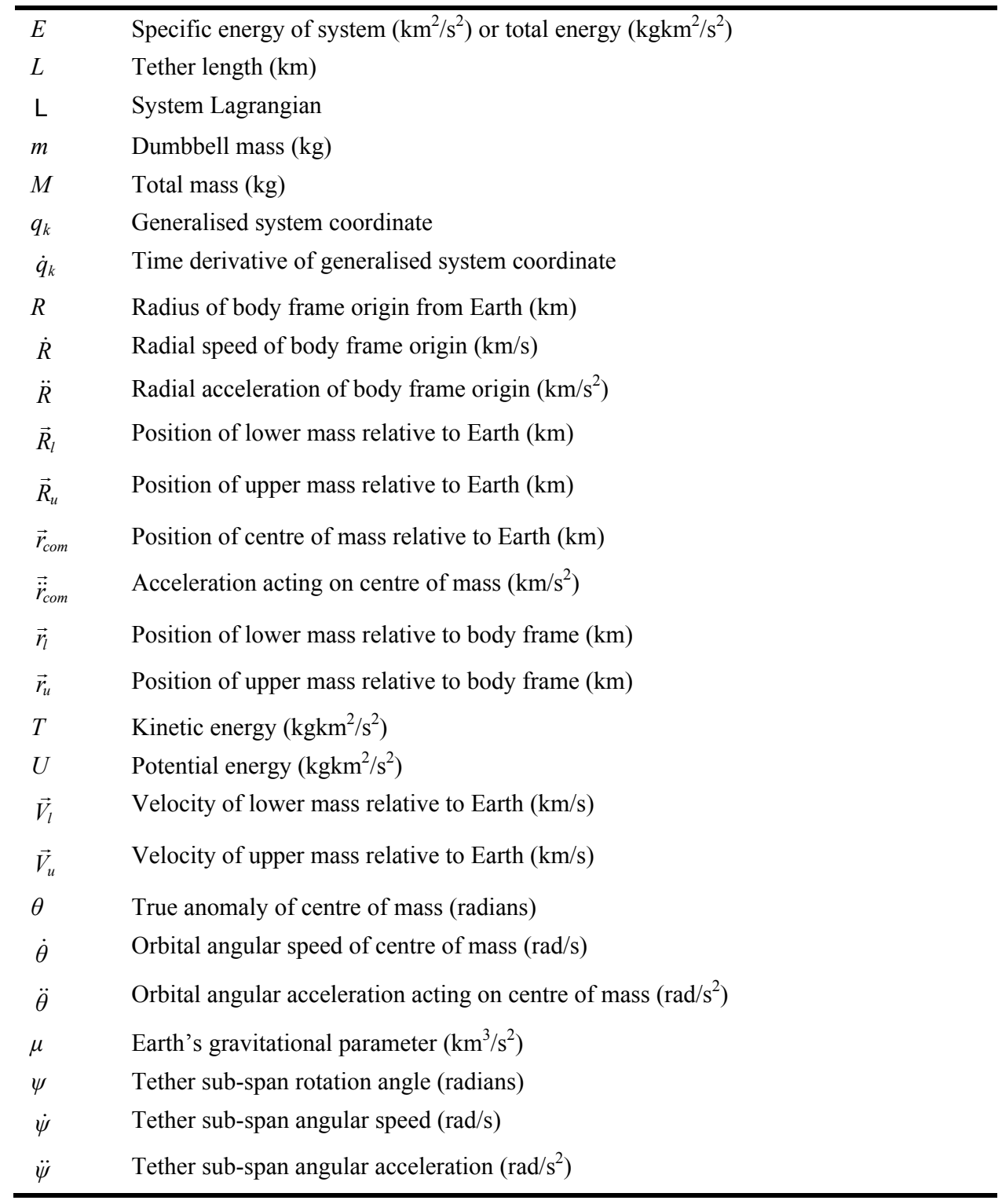

